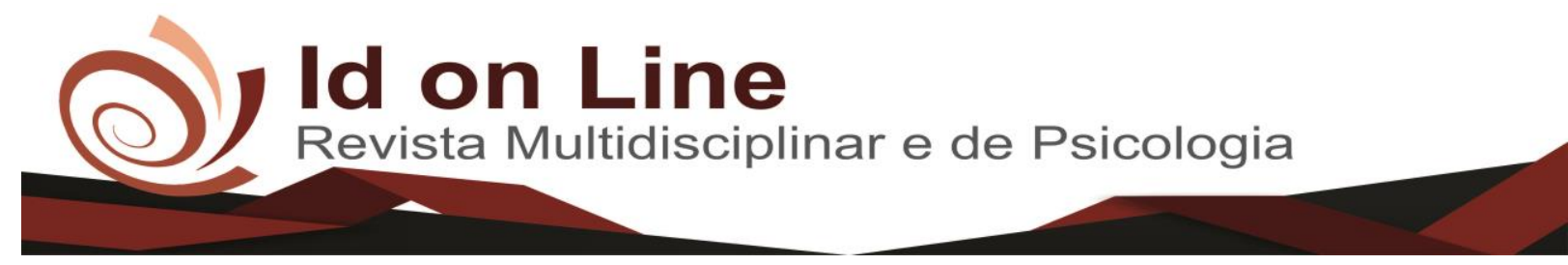

Artigo

\title{
Caracterização da Maturidade Perceptivo-Motora nas Crianças com Fissura de Lábio e Palato
}

\author{
Carolina Tarcinalli Souza ${ }^{l}$; Mariana de Freitas Pereira ${ }^{2}$; Maria de Lourdes Merighi Tabaquim ${ }^{3}$
}

\begin{abstract}
Resumo: O desenvolvimento motor é organizado a partir do início da concepção, seguindo os princípios do domínio motor, afetivo-social e cognitivo, que vão se diferenciando gradativamente. O resultado da interação de todos esses fatores após o nascimento é expresso pelo comportamento motor, indicando sua importância no desenvolvimento do ser humano. O objetivo deste estudo foi caracterizar a maturidade perceptivo-motora da dominância lateral, esquema corporal, orientação espaço-temporal e motricidade fina de crianças com fissura labiopalatina, comparadas ao grupo controle. A amostra foi composta por 102 participantes, de ambos os gêneros, idade entre 8 a 12 anos, sendo GI como grupo referência, com 51 sujeitos, ambos os sexos, matriculados no ensino regular, e GII, como grupo comparativo, composto por 51 participantes com diagnóstico de fissura labiopalatina, sem síndrome e alteração neurológica. Na comparação entre GI e GII, encontrou-se diferença estatisticamente significante na praxia global, com escores abaixo da média esperada. No entanto, ambos os grupos tiveram performances satisfatórias quanto à tonicidade, equilíbrio, lateralidade, noção e estruturação. Assim este estudo apontou discrepâncias descritivas, no perfil psicomotor de ambos os grupos.
\end{abstract}

Palavras-chave: Desenvolvimento motor. Fissura palatina. Maturidade perceptivo-motora.

\section{Characterization of Perceptive-Motor Maturity in Children with Lips and Palato Fissure}

\begin{abstract}
The motor development is organized from the beginning of the conception, having followed the principles of motor, affective-social and cognitive the domain, that gradual go if differentiating. The result of the interaction of all these factors after the birth is express for the motor behavior, indicating its importance in the development of the human being. The objective of this study to characterize the perceptive-motor maturity of lateral dominance, body schema, space-temporal orientation and fine motor skills of children with cleft lip and palate, compared to the control group. The sample was composed for 102 participants, of both the sorts, age enters 812 years, being GI as group reference, with 51 citizens, both the sex, registered regular education, and GII, as comparative, composed group for 51 participants with diagnosis of cleft palate fiction, without syndrome and neurological alteration. In the process of validation of the instrument, it had adjustments how much to the vernaculars of the Brazilian Portuguese, referring to the idiomatic and semantic translations, as well as in the instructional statements of the tests of the instrument. In the comparison between GI and GII, significant difference in the gross praxis met statistical, with props up below of the waited average. However, both the groups had had satisfactory performances how much to the equilibrium, lateralization, body perception, gross and fine praxis, tonus and time-space orientation. This study pointed to descripitive discrepancies in the phychmotor profile of both groups.
\end{abstract}

Keywords: Motor development. Cleft palate. Perceptive-motor maturity.

\footnotetext{
${ }^{1}$ Docente na Faculdades Integradas de Bauru, Doutora em Ciências da Reabilitação. Bauru, S.P - Brasil.

${ }^{2}$ Mestranda em Psicologia do Desenvolvimento e aprendizagem pela UNESP. Bauru, S.P, Brasil

${ }^{3}$ Doutora em Ciências Médicas. Docente da Faculdade de Odontologia de Bauru-SP, Brasil.

Autor Correspondente: Carolina Tarcinalli Souza Rua São Gonçalo 6-73; CEP:17012-170; Bauru/SP Fone (14) 981387767 Email: caroltar@msn.com
} 


\section{Introdução}

O desenvolvimento craniofacial normal dos vertebrados é um evento complexo e dinâmico, o qual envolve múltiplos estágios do desenvolvimento, iniciando com a formação da crista neural, necessária ao desenvolvimento do sistema nervoso central. O sistema nervoso central (SNC), fundamental para o desenvolvimento do complexo craniofacial, surge da placa neural, uma camada homogênea de células epiteliais que constituem a superfície dorsal no estágio de gástrula. À medida que a placa neural se dobra para formar o tubo neural, o final anterior alargado se segmenta em três vesículas. Essas vesículas são os primórdios do desenvolvimento do cérebro anterior (prosencéfalo), cérebro médio (mesencéfalo) e cérebro posterior (rombencéfalo). A expansão do cérebro anterior dá origem ao processo frontonasal e, as células da crista neural, derivadas do cérebro médio e do cérebro posterior (que, nesse momento, encontra-se segmentado em 8 pares de rombômeros), migram para a região dos arcos faríngeos, dando origem à maioria do mesênquima dos arcos faríngeos, os quais estão intimamente relacionados ao desenvolvimento das regiões média e baixa do complexo craniofacial (CHAI et al., 2000; WILKIE e MORRIS-KAY, 2001; MAO e NAH, 2004). Dessa forma, o ponto central do desenvolvimento craniofacial é a segmentação do SNC que, em conjunto com a migração das células da crista neural, dão origem à grande parte da cabeça e do pescoço e de seus compartimentos associados e individualizados.

A disrupção na regulação do processo de desenvolvimento craniofacial, causado por fatores genéticos e/ou ambientais, pode resultar em diferentes tipos de anomalias craniofaciais, as quais incluem, principalmente, alterações estruturais de SNC, defeitos de fechamento dos ossos do crânio, defeitos de linha média craniofacial, fissuras orofaciais típicas e atípicas, e alterações de primeiro e segundo arcos faríngeos (WILKIE; MORRIS-KAY, 2001). Dentre as malformações determinadas por alterações da morfogênese encontram-se as fissuras labiopalatinas, as quais apresentam sua etiologia de herança multifatorial, sendo o fator hereditário identificado em cerca de 35\% e 65\% associado à ação ambiental (MANOEL, 2006).

As alterações morfológicas em indivíduos com fissura labiopalatina, além de resultar interferência na estruturas e em algumas funções, comprometem tanto a estética quanto a eficiência de vários sistemas, dentre eles, o estomatognático, que interferem no processo de 
comunicação, com danos para a aprendizagem e interação social, assim como, no desenvolvimento de competências cognitivas e afetivas (MCARTHUR e BISHOP, 2004).

Entretanto para que desenvolvam as habilidades percepto-motoras é importante a integridade do Sistema Nervoso Central e Sistema Nervoso Periférico resultando no desenvolvimento neuropsicomotor normal. O desenvolvimento motor está intimamente relacionado ao desenvolvimento do sistema perceptivo (MEDINA; ROSA; MARQUES, 2006). A percepção resulta em estímulos sensoriais, que desencadeiam uma ação, a habilidade cognitiva ou motora, sendo assim, problemas na percepção pode afetar o aprendizado de habilidades motoras (COUTINHO; SOUZA, 2014).

O desenvolvimento perceptivo-motor decorre da organização de novas informações somadas às já existentes, imprescindível para o movimento eficaz. O processo perceptivo-motor ocorre em diferentes fases, tais como, o estímulo, a recepção de informações ambientais, o processamento, a escolha adequada e a realização do movimento (GALLAHUE; OZMUN, 2005). Por exemplo, quando um estímulo (ex.: uma bola lançada) é dado, as informações sensoriais do ambiente são coletadas pelos receptores especializados (ex.: tátil, auditivo) e são transmitidos ao cérebro como energia neural. Lá ocorre o processamento e a comparação da informação recebida com a informação já armazenada anteriormente, possibilitando, então, a escolha da melhor opção de movimento. Quando o movimento é realizado, acontece o feedback por meio de receptores especializados para a informação ser moldada ou armazenada (PAYNE; ISAACS, 2007).

Para Pinheiro, Martinez, Fontaine (2014), a integração viso motora é definida como o grau em que a percepção visual e os movimentos das mãos e dedos estão coordenados. Porém, caso não sejam executados de forma coordenada, surgem as disfunções psicomotoras, prejudicando o desempenho da criança no seu papel ocupacional na realização de diversas atividades de vida diária, como comer, no auto-cuidado e no lazer. Também se constata impacto nas atividades acadêmicas, como observado em cópia de figuras, recorte e escrita, entre outras.

Tabaquim, Ferrari e Souza (2015) investigaram a percepção visual de crianças com fissura labiopalatina não sindrômica e sem prejuízo intelectual, com idade entre 10 anos e 10 anos e 11 meses, por meio da avaliação neuropsicológica, identificaram que aquelas com o tipo transforame foram as mais prejudicadas nas funções visomotoras, com domínios perceptivos construtivos classificados abaixo da média esperada para a faixa etária. 
Conrad et al. (2009), realizaram avaliação neuropsicológica em crianças com e sem fissura labiopalatina não sindrômicas, com idade entre 7 e 17 anos de ambos os sexos, encontrando alterações nas funções verbais e de memória nos participantes com fissuras labiopalatinas.

Apesar da criança com fissura labiopalatina apresentar prejuízos nas habilidades cognitivas, alterações nas funções auditivas e /ou na fala que são essenciais para o processo de aprendizagem (PRUDENCIATTI; ROCHA DE VASCONCELLOS; TABAQUIM, 2017) existe uma escassez de pesquisas que avaliam a habilidades perceptivo-motoras. Dessa maneira o objetivo deste presente artigo foi caracterizar a maturidade perceptivo-motora da dominância lateral, esquema corporal, orientação espaço-temporal e motricidade fina de crianças com fissura labiopalatina, comparadas ao grupo controle.

\section{Método}

Fizeram parte deste estudo comparativo de validação 100 participantes, ambos os sexos, na idade de 8 a 12 anos, cursando o ensino fundamental. Os participantes compuseram dois grupos $^{1}$ :

GI: 50 participantes sem fissura, regularmente matriculados na rede pública de escolas brasileiras.

GII: 50 participantes com fissura labiopalatina, inscritas no Hospital de Reabilitação de Anomalias Craniofaciais, Universidade de São Paulo (HRAC-USP).

No planejamento amostral foram considerados o poder de $80 \%$ e $\alpha 5 \%$, desvio padrão 0,84 e uma diferença mínima a ser detectada de 0.5 na amostra, por ser este um instrumento paramétrico do presente estudo.

\footnotetext{
${ }^{1}$ Pesquisas sobre adaptação de instrumentos têm recebido grande ênfase na comparação de resultados por meio de estudos realizados em amostras distintas

Hambleton. Issues, designs, and technical guidelines for adapting tests into multiple languages and cultures. Adapting educational and psychological tests for cross-cultural assessment.2005: 3-38,Gjersing L, Caplehorn JRM, Clausen T. Cross-cultural adaptation of research instruments: language, setting, time and statistical considerations. BMC Med Res Methodol. 2010;10:13.
} 


\section{Critérios de inclusão:}

GI: estar matriculado na rede oficial de ensino público do ensino fundamental; estar na faixa etária de 08 a 12 anos de idade; e apresentar o consentimento formal para a participação na pesquisa por meio do Termo de Consentimento Livre e Esclarecido (TCLE). Anexo A

GII: estar inscrito no programa de atendimento do HRAC-USP; ter o diagnóstico de fissura labiopalatina; estar na faixa etária proposta no estudo; estar matriculado na rede oficial de ensino público do ensino fundamental; e, consentir formalmente na participação na pesquisa por meio do TCLE.

\section{Critérios de Exclusão:}

GI e GII: apresentar prejuízo intelectual (nível V do instrumento de triagem da pesquisa Matrizes Progressivas Coloridas), relatos de alterações neurológicas, psiquiátricas, sensoriais ou sindrômicas, decorrentes de informações de prontuário clínico hospitalar e acadêmico.

\section{Procedimentos}

A coleta de dados do GI foi realizada na escola regular de ensino fundamental EEPG Prof ${ }^{a}$ Mercedes Paes Bueno, já o GII foi constituído por usuários do Hospital de Reabilitação de Anomalias Craniofaciais da Universidade de São Paulo (HRAC-USP). Os dados foram coletados no Laboratório de Neuropsicologia do HRAC-USP.

Os instrumentos utilizados foram os seguintes: Entrevista Estruturada que foi construída para este estudo, o protocolo de entrevista teve o objetivo de caracterizar a amostra, considerando as variáveis: idade, gênero, escolaridade, e nível socioeconômico, (APÊNDICE A) atendendo aos oito critérios da Secretaria de Assuntos Estratégicos para renda familiar (SAE, 2012), de acordo com os níveis: (1) extremamente pobre; (2) pobre, mas não extremamente pobre; (3) vulnerável; (4) baixa classe média; (5) média classe média; (6) alta classe média; (7) baixa classe alta; (8) alta classe alta (ANEXO C). O Teste Matrizes Progressivas Coloridas que é constituído por três séries de 12 itens: $\mathrm{A}, \mathrm{Ab}$ e $\mathrm{B}$, dispostos em ordem de dificuldade crescente, cujo 
objetivo é avaliar o nível intelectual e o raciocínio espaço-temporal lógico. O instrumento é composto por 30 matrizes, em que falta uma parte no conjunto. O desenho faltante é apresentado entre seis alternativas, uma das quais completa a matriz corretamente, e deverá ser identificada pelo examinando (PASQUALI; WECHSLER; BENSUSAN, 2002).

Outro instrumento utilizado foi a Bateria Psicomotora (BPM) composta de sete fatores psicomotores: tonicidade, equilíbrio, lateralidade, noção do corpo, estruturação espaçotemporal, praxia global e praxia fina, subdivididos em 26 sub-fatores. A bateria apresenta oportunidades para o estudo da psicomotricidade atípica em pessoas com diferentes deficiências, ou seja, físicas, sensoriais, da comunicação e sócio-emocionais. São 42 tarefas distribuídas entre os fatores que permitem classificar em quatro perfis psicomotores: 1 . Realização imperfeita, incompleta e descoordenada (fraco) - perfil apráxico; 2. Realização com dificuldades de controle (satisfatório) - perfil dispráxico; 3. Realização controlada e adequada (bom) - perfil eupráxico; 4. Realização perfeita, econômica, harmoniosa e bem controlada (excelente) - perfil hiperpráxico. O perfil psicomotor final dos fatores será a soma das pontuações das tarefas e a divisão pelo número de tarefas de cada fator. O resultado será obtido cotando os quatro dos sub-fatores que traduz em de forma global o respectivo perfil psicomotor (FONSECA, 2012).

\section{Resultados}

Tabela 1. Caracterização demográfica dos grupos de estudo.

\begin{tabular}{|c|c|c|c|c|c|}
\hline \multirow[b]{2}{*}{ Variáveis } & & GI & \multicolumn{2}{|l|}{ GII } & p-valor \\
\hline & & Média $\pm D P$ & \multicolumn{3}{|l|}{ Média $\pm D P$} \\
\hline Idade* $^{*}$ & & $9,9 \pm 1,4$ & $10,5 \pm 1,3$ & & 0,0375 \\
\hline Pontos* & & $24,0 \pm 3,4$ & $24,6 \pm 2,7$ & & 0,3348 \\
\hline \multirow[t]{2}{*}{ Média* } & & $3,4 \pm 0,5$ & $3,5 \pm 0,4$ & & 0,4553 \\
\hline & & $\mathrm{n}(\%)$ & $\mathrm{n}(\%)$ & Total & \\
\hline \multirow[t]{2}{*}{ Sexo** } & $\mathrm{F}$ & $33(64,7)$ & $21(41,2)$ & 54 & 0,0173 \\
\hline & $\mathrm{M}$ & $18(35,3)$ & $30(58,8)$ & 48 & \\
\hline
\end{tabular}

Continua... 


\begin{tabular}{llllll}
\hline Escolaridade & Até $4^{\text {a }}$ série & $29(56,9)$ & $21(41,2)$ & 50 & 0,1131 \\
& $5^{\text {a }}$ série e mais & $22(43,2)$ & $30(58,8)$ & & \\
& Normal & $14(27,5)$ & $4(7,8)$ & 18 & 0,0246 \\
Bom & $22(43,1)$ & $32(62,8)$ & 54 & \\
Classificação** & $15(29,4)$ & $15(29,4)$ & 30 & \\
& Superior & & & & \\
Nivel (Haven)** & & & & \\
& I & $2(3,9)$ & $5(9,9)$ & 7 & 0,3090 \\
& II & $24(47,1)$ & $22(43,1)$ & 46 & \\
III & $19(37,2)$ & $22(43,1)$ & 41 & \\
IV & $6(11,8)$ & $2(3,9)$ & 8 & \\
Condição socioeconômica** & Baixo & $19(37,3)$ & $5(9,8)$ & 24 & 0,0039 \\
& Médio-Baixo & $9(17,6)$ & $10(19,6)$ & 19 & \\
Médio & $23(45,1)$ & $36(70,6)$ & 59 & \\
\hline
\end{tabular}

*-teste t-Student; $* *$-teste qui-quadrado

Os resultados da avaliação intelectual da amostra, utilizando as Matrizes Progressivas Coloridas, demonstraram que no GI 2 (3,9\%) encontraram-se no nível I (Superior), no GII 5 (9,8\%). O GI no nível II (acima da média) encontrou-se com 24 (47,1\%) e GII 22 (43,1\%). Para o nível III (médio) o grupo GI apresentou 19 (37,3\%) e o grupo GII $22(43,1 \%)$. E com relação ao nível IV intelectual (abaixo) o grupo GI encontrou-se com 6 (11,8\%) e grupo GII 2 (3,9\%) (Tabela 1). Quanto ao tempo de execução, relacionado à velocidade de processamento visual, não houve diferença significante entre os grupos nesta tarefa intelectual.

Na Bateria Psicomotora verificou-se a qualificação psicomotora dos 102 participantes, no qual $18(17,64 \%)$ estavam dentro da normalidade, $30(29,41 \%)$ superior e $54(52,94 \%)$ bom.

Avaliando a Bateria Psicomotora por grupo (Tabela 2) foi possível observar que, quanto à tonicidade, a média dos participantes tanto do GI quanto do GII foi de 4,0 pontos, estando próximo ao valor recomendado pelo instrumento. Já quanto ao equilíbrio a média encontrada no GI e no GII foi de 3,4 pontos, estando também próximo à recomendação. Analisando a lateralidade o mesmo resultado foi encontrado, estando o GI com uma média de 4,0 pontos e o GII 3,9 atingindo resultado esperado. Quanto à noção e estruturação o mesmo resultado foi encontrado. $\mathrm{Na}$ análise da praxia fina o resultado foi abaixo do esperado do critério do 
instrumento para ambos os grupos, entretanto, na classificação geral da Bateria Psicomotora foi tida como "bom".

Tabela 2. Comparação das médias da Bateria Psicomotora por grupo

\begin{tabular}{l|l|l|l|l|l|l|l|l}
\hline \multicolumn{1}{c|}{ Grupo } & Tonicidade & Equilíbrio & Lateralidade & Noção & $\begin{array}{c}\text { Estrutu- } \\
\text { ração }\end{array}$ & $\begin{array}{c}\text { Praxia } \\
\text { Global }\end{array}$ & $\begin{array}{c}\text { Praxia } \\
\text { Fina }\end{array}$ & $\begin{array}{c}\text { Classi- } \\
\text { ficação }\end{array}$ \\
\hline GI & $4,0 \pm 0,1$ & $3,4 \pm 0,6$ & $4,0 \pm 0,0$ & $3,5 \pm 0,8$ & $3,3 \pm 1,0$ & $3,6 \pm 0.5$ & $2,3 \pm 1,4$ & BOM \\
\hline GII & $4,0 \pm 0,1$ & $3,4 \pm 0,8$ & $3,9 \pm 0,4$ & $3,5 \pm 1,0$ & $3,5 \pm 0,9$ & $3,9 \pm 0,3$ & $2,2 \pm 1,4$ & BOM \\
\hline p-valor* & 1,0000 & 0,8895 & 0,0552 & 0,8267 & 0,1939 & 0,0006 & 0,9447 & \\
\hline
\end{tabular}

Obs: Máximo a ser atingido na bateria é 4,0 pontos. *- teste t-Student

\section{Discussão}

A avaliação psicomotora, proposta na Bateria de Psicomotora (FONSECA, 2013), envolve aspectos como maturação biológica, desenvolvimento cognitivo, integração visomotora, entre outros, fundamental para auxiliar na definição de quadros clínicos na infância, em diferentes populações. Cabe ao profissional, não só estabelecer o perfil das fragilidades e sua extensão funcional, mas também as habilidades preservadas, potenciais.

Na comparação amostral, foi elegido o grupo de crianças com fissura labiopalatina isolada, por apresentarem maior vulnerabilidade para distorções na interpretação dos sinais sensoriais no processamento das percepções (FENIMAN, SOUZA, TEIXEIRA, MONDELI, 2012). Essas alterações são passíveis de interferirem nas habilidades essenciais dos processos de aprendizagem, como as alterações de fala e de organização psicomotora (TABAQUIM, FERRARI, SOUZA, 2015).

Na caracterização do fator sócio econômico, foi verificada na amostra total, maior incidência na classificação média, porém, o GI como grupo de referência, evidenciou condições socioeconômicas em níveis médio-baixo e baixo. Este dado mostra-se relevante ao considerar este grupo com nível intelectual inferior quando comparado ao GII, com fissura labiopalatina. Desta forma, uma análise mais cuidadosa sobre as condições sociodemográficas da amostra, aponta para os fatores de risco elevados e de proteção diminuídos do GI, com prejuízos na qualidade de vida e 
alta prevalência de problemas de comportamento motivacional para a aprendizagem (MARTINI, PADOVANI, PEROSA, 2016), sinalizando para a necessidade de serviços de acompanhamento no ingresso da criança na escola.

Os resultados da avaliação intelectual da presente amostra, utilizando as Matrizes Progressivas Coloridas, evidenciaram no GII um perfil com maiores recursos de raciocínio não verbal, com capacidade para estabelecer analogias, relações de causa e efeito e articular o pensamento lógico abstrato, condições estas essenciais para o bom desempenho acadêmico. As pesquisas apontam (MECCA; JANA; SIMÕES; MACEDO, 2015) para uma maior influência das variáveis socioeconômicas nas medidas verbais em relação às não verbais, porém, nossos resultados indicaram prejuízos em tarefas cognitivas não verbais na amostra composta por participantes com nível mais deficitário.

Sendo assim, os pais com melhores condições socioeconômicas proporcionam melhores oportunidades e qualidade de vida, contribuindo para melhores habilidades cognitivas, constatado pelo desempenho das crianças em tarefas tanto verbais quanto não verbais. Por outro lado, as crianças com baixo nível socioeconômico apresentam uma capacidade mais limitada em tarefas acadêmicas e em relação às suas próprias habilidades cognitivas, assim como, à população geral. (ARDILA ET AL., 2005, CATALE, WILLEMS, LEJEUNE, \& MEULEMANS, 2012; PALEARI, TABAQUIM, 2013; JACOBSEN, MORAES WAGNER , TRENTINI 2013). Isso foi observado no estudo de Souza et al., (2015) onde verificou-se que os grupos de menor nível socioeconômico apresentaram diferenças estatísticas para provas da coordenação motora grossa, relacionadas á força muscular. Isso pode ser justificado por Versan (2010) quando afirma que o nível de coordenação motora de crianças está relacionado com as condições socioeconômicas e ambientais em que vivem, além, das oportunidades de acesso à saúde, à educação, à alimentação adequada e a prática de atividades físicas necessárias ao desenvolvimento da coordenação motora global. Em um estudo com escolares de 6 a 10 anos, foi mostrado que $96 \%$ dos escolares apresentaram classificação de coordenação motora normal, $1 \%$ para coordenação motora acima da normalidade e $3 \%$ abaixo da normalidade (ROSA NETO et al., 2010).

No presente estudo, $72 \%$ das meninas do grupo Cidade apresentaram coordenação motora normal, $25 \%$ com perturbações na coordenação motora e 3\% insuficiente. Já para os meninos desse mesmo grupo, $78 \%$ tiveram coordenação motora normal, $16 \%$ perturbações, iguais 3\% para insuficiente e boa. Quanto ao grupo Vila, 72\% das meninas apresentaram 
coordenação motora normal, $21 \%$ perturbação e $7 \%$ boa. No entanto, para os meninos deste grupo, $71 \%$ apresentaram coordenação normal, $13 \%$ perturbação, $8 \%$ insuficiente e $13 \%$ boa. Esses resultados mostram que no estudo de Rosa Neto et al., (2010) quase a totalidade dos escolares apresentaram coordenação motora normal, o que não ocorreu com os grupos da pesquisa hora apresentada, isso também pode estar associado ao nível socioeconômico e a diferença regional entre as pesquisas

$\mathrm{Na}$ realização das tarefas acadêmicas, o desenvolvimento psicomotor é marcado pela evolução dos movimentos, do mais simples ao mais complexo, do global ao refinado, e do difuso ao seletivo. Neste estudo, verificou-se que o GII obteve melhor resultado na praxia global com relação ao GI, corroborando com o estudo de Campos et al., (2008), que investigou habilidades práxicas globais e finas de crianças do ensino fundamental e identificou recursos predominantemente dispráxicos.

A carência de aulas de educação física na escola e de propostas pedagógicas para promover o desenvolvimento de habilidades motoras tem sido apontada como contribuinte para os atrasos na população de crianças do ensino fundamental (NOBRE, BANDEIRA, VALENTINI, 2016). Os microssistemas escolares, juntamente com os programas sociais são influenciados pela omissão do poder público (exo e macrosistemas), repercutindo no desempenho dos escolares.

Neste estudo, não foi possível contemplar a análise de todos os componentes cognitivos e psicomotores envolvidos na avaliação, tendo sido mantidos aqueles considerados essenciais para um exame breve em sessão única. Para complementar o estudo diagnóstico funcional, sugere-se o uso de outros instrumentos complementares, mais específicos, para uma investigação mais aprofundada. Mesmo em face dessas limitações, o desenvolvimento criterioso das adequações instrucionais desta ferramenta de investigação psicomotora infantil, deverá contribuir para suprir a escassez de instrumentos nacionais padronizados de avaliação dos processos neuropsicomotores nesta faixa de desenvolvimento, assim como, contribuir como auxiliar no processo de criação de outros protocolos. 


\section{Conclusão}

Concluiu-se que na maturidade perceptivo-motora não houve diferença significante entre os grupos GI e GII quanto a tonicidade, equilíbrio, lateralidade, Noção, Estruturação, porém o GII ao ser avaliado descritivamente apresentou melhores habilidades motoras dentro da praxia global.

Os resultados apontaram discrepâncias descritivas, considerando o perfil psicomotor de desempenhos.

\section{Referências}

ARDILA, Alfredo et al. The influence of the parents' educational level on the development of executive functions. Developmental neuropsychology, v. 28, n. 1, p. 539-560, 2005.

CAMPOS, Ana Carolina de et al. Psychomotor intervention on children of low socioeconomic status. Fisioterapia e Pesquisa, v. 15, n. 2, p. 188-193, 2008.

CATALE, Corinne et al. Parental educational level influence on memory and executive performance in children. Revue Européenne de Psychologie Appliquée/European Review of Applied Psychology, v. 62, n. 3, p. 161-171, 2012.

CHAI, Yang et al. Fate of the mammalian cranial neural crest during tooth and mandibular morphogenesis. Development, v. 127, n. 8, p. 1671-1679, 2000.

CONRAD, Amy Lynn et al. Neuropsychological functioning in children with non-syndromic cleft of the lip and/or palate. Child Neuropsychology, v. 15, n. 5, p. 471-484, 2009.

COUTINHO, Mônia Tainá Cambruzzi; SOUZA, Mariele Santayana de. Percepção na infância: conceitos e aplicações práticas em aulas de Educação Física. Disponível em: .Acesso em: 24 de agosto de 2017

COUTO, Renata Cardoso; LEAL, Flávia de Jesus; PITTA, Guilherme Benjamin Brandão. Validação do questionário de qualidade de vida na úlcera venosa crônica em língua portuguesa (Charing Cross Venous Ulcer Questionnaire-CCVUQ-Brasil). J Vasc Bras, v. 15, n. 1, p. 4-10, 2016.

FENIMAN, Mariza Ribeiro et al. Perception of parents about the auditory attention skills of his kid with cleft lip and palate: retrospective study. Arquivos Internacionais de Otorrinolaringologia, v. 16, n. 1, p. 115-120, 2012.

FONSECA V. Desenvolvimento psicomotor e aprendizagem. Porto Alegre: Artmed, 2008. 
Manual de observação psicomotora: significação psiconeurológica dos fatores psicomotores. Rio de Janeiro: Wak; 2012.

GALLAHUE DL, OZMU JC, GOODWAY JD. Compreendendo o desenvolvimento motor: bebês, crianças, adolescentes e adultos. São Paulo: Phorte; 2003.

JACOBSEN, Geise M. et al. Qual é a participação de fatores socioeconômicos na inteligência de crianças?. Revista Neuropsicologia Latinoamericana, v. 5, n. 4, p. 32-39, 2013.

MANOEL Rosana R. O comportamento auditivo do aluno com fissura labiopalatina: o julgamento do professor (tese), 144f Bauru: Hospital de Reabilitação de Anomalias Craniofaciais, Universidade de São Paulo, 2006.

MARTINI, Juliana Aparecida; PADOVANI, Flávia Helena Pereira; PEROSA, Gimol Benzaquen. Quality of life of preterm children: risk and protective factors. Paidéia (Ribeirão Preto), v. 26, n. 65, p. 325-332, 2016.

MCARTHUR, G. M.; BISHOP, D. V. M. Which people with specific language impairment have auditory processing deficits?. Cognitive Neuropsychology, v. 21, n. 1, p. 79-94, 2004.

PONTRELLI MECCA, Tatiana et al. Relação entre habilidades cognitivas não-verbais e variáveis presentes no contexto educacional. Psicologia Escolar e Educacional, v. 19, n. 2, 2015.

MEDINA, Josiane; BROIO ROSA, Greisy. K. B., MARQUES, Inara. Desenvolvimento da organização temporal de crianças com dificuldades de aprendizagem. Revista da Educação Física/UEM, Maringá, v. 17, n. 1, p. 107-116, 2006.

CARVALHO NOBRE, Glauber; RIBEIRO BANDEIRA, Paulo Felipe; CRISTINA VALENTINI, Nadia. Relação entre a competência motora percebida geral, o gênero, a competência motora percebida específica à tarefa e a competência motora real de crianças. Journal of Physical Education, v. 27, n. 1, p. e2744, 29 mar. 2016

PASQUALI, Luiz. Psychometrics. Revista da Escola de Enfermagem da USP, v. 43, n. SPE, p. $992-$ 999, 2009.

PAYNE, V. G., ISAACS, L. D. Desenvolvimento motor humano: uma abordagem vitalícia. $6^{\mathrm{a}}$ edição. Rio de Janeiro: Guanabara Koogan, 2007

PINHEIRO, Raquel Cristina; MARTINEZ, Claudia Maria Simões; FONTAINE, Anne Marie Germaine Victorine. Integração viso motora e desenvolvimento global de crianças pré-termo e a termo no início da escolarização. Rev. bras. crescimento desenvolv. hum., São Paulo , v. 24, n. 2, p. 181-187, 2014 - Disponível em <http://pepsic.bvsalud.org/scielo.php?script=sci_arttext\&pid=S010412822014000200010\&lng=pt\&nrm=iso > acessos em 05 jul. 2018.

PRUDENCIATTI, Shaday; HAGE, Simone Rocha de Vasconcellos; TABAQUIM, Maria de Lourdes Merighi. Desempenho cognitivo-linguístico de crianças com fissura labiopalatina em fase de aquisição da leitura e escrita. Rev. CEFAC, São Paulo, v. 19, n. 1, p. 20-26, Feb. 2017 . Available from $<$ http://www.scielo.br/scielo.php?script=sci_arttext\&pid=S1516-

$18462017000100020 \& \operatorname{lng}=$ en\&nrm=iso>. access on 05 July 2018. http://dx.doi.org/10.1590/19820216201719110416. 
ROSA NETO, F. et al. Importância da avaliação motora em escolares: análise da confiabilidade da Escala de Desenvolvimento Motor. Revista Brasileira de Cineantropometria e Desempenho Humano, Florianópolis, v. 12, n. 6, p. 422-427, 2010.

SOUZA, Rosiane de Nazaré da Silva de et al. NÍVEL SOCIOECONÔMICO, ESTADO NUTRICIONAL E COORDENAÇÃO MOTORA GROSSA DE ESCOLARES COM 6 A 10 ANOS NA AMAZÔNIA. Rev. educ. fis. UEM, Maringá , v. 26, n. 3, p. 401-411, Sept. 2015. Available from <http://www.scielo.br/scielo.php?script=sci_arttext\&pid=S1983$30832015000300401 \& \operatorname{lng}=$ en\&nrm=iso $>$.access

on 05 July 2018. http://dx.doi.org/10.4025/reveducfis.v26i3.26212.

TABAQUIM, Maria de Lourdes Merighi et al. Visual-constructive dyspraxia of children with cleft lip and palate. International Journal of Humanieties and Social Science, v. 4, n. 7, p. 1, 2014.

TABAQUIM, Maria de Lourdes Merighi; FERRARI, Juliana Brocco; SOUZA, Carolina Tarcinalli. Funções percepto-motoras de crianças com fissura labiopalatina. Revista Brasileira em Promoção da Saúde, v. 28, n. 1, p. 89-97, 2015.

VERSAN, D. T. Estudo sobre o impacto socioambiental no desempenho motor de crianças de 7 a 8 anos - região noroeste de Goiânia/GO. 2010. Dissertação (Mestrado em Ciências Ambientais e Saúde) Pontifícia Universidade Católica de Goiás, Goiânia, 2010.

WELCH S, COMER J. Quantitative methods for public administration: techniques and applications. Virginia:Brooks; 2001.

WILKIE, Andrew OM; MORRISS-KAY, Gillian M. Genetics of craniofacial development and malformation. Nature Reviews Genetics, v. 2, n. 6, p. 458, 2001.

\section{Como citar este artigo (Formato ABNT):}

SOUZA, Carolina Tarcinalli; PEREIRA, Mariana de Freitas; TABAQUIM, Maria de Lourdes Merighi. Caracterização da Maturidade Perceptivo-Motora nas Crianças com Fissura de Lábio e Palato. Id on Line Rev.Mult. Psic., 2018, vol.12, n.41, p.570-582. ISSN: 1981-1179.

Recebido: 05/07/2018

Aceito 07/07/2018 